\title{
Response of the QT interval to mental and physical stress in types LQT1 and LQT2 of the long QT syndrome
}

\author{
K J Paavonen, H Swan, K Piippo, L Hokkanen, P Laitinen, M Viitasalo, L Toivonen, \\ K Kontula
}

\begin{abstract}
Objective-To study and compare the effects of mental and physical stress on long QT syndrome (LQTS) patients.

Design-Case-control study.

Main outcome measures-QT intervals were measured from lead V3. Serum potassium and plasma catecholamine concentrations were also monitored.

Patients-16 patients with type 1 LQTS (LQT1), 14 with type 2 LQTS (LQT2), both groups asymptomatic, and 14 healthy control subjects.

Interventions-Three types of mental stress tests and a submaximal exercise stress test.

Results-Heart rate responses to mental stress and exercise were similar in all groups. During mental stress, the mean QT interval shortened to a similar extent in controls $(-29 \mathrm{~ms})$, LQT1 patients $(-34 \mathrm{~ms})$, and LQT2 patients $(-30 \mathrm{~ms})$. During exercise, the corresponding QT adaptation to exercise stress was more pronounced $(\mathrm{p}<0.01)$ in healthy controls $(-47 \mathrm{~ms})$ than in LQT1 $(-38 \mathrm{~ms})$ or LQT2 patients $(-38 \mathrm{~ms})$. During exercise changes in serum potassium concentrations were correlated to changes in QT intervals in controls, but not in LQTS patients. LQT1 and LQT2 patients did not differ in serum potassium, catecholamine or heart rate responses to mental or physical stress.

Conclusions-QT adaptation to mental and exercise stress in healthy people and in patients with LQTS is different. In healthy people QT adaptation is more sensitive to physical than to mental stress while no such diverging pattern was seen in asymptomatic LQTS patients.

(Heart 2001;86:39-44)
\end{abstract}

Keywords: exercise; long QT syndrome; mental stress; potassium channel

The inherited long QT syndrome (LQTS) is a myocardial ion channel disease characterised by prolongation of the QT interval in the ECG associated with a predisposition to ventricular arrhythmias and sudden death. ${ }^{1-4}$ The congenital LQTS can be classified into types LQT1, LQT2, LQT3, and LQT5 according to the underlying gene defect, which may involve a mutation of the potassium channel subunits KCNQ1 or HERG, the sodium channel SCN5A, or the minimal potassium channel subunit MinK, respectively. An as yet unidentified LQTS gene may reside on chromosome 4 (LQT4) and additional rare forms of LQTS may be caused by mutations of the KCNE2 gene encoding the MinK related potassium channel subunit (LQT6). ${ }^{5}$ Triggers of cardiac events may vary from type to type: in LQT1 caused by KCNQ1 mutations, physical exercise typically provokes arrhythmias, while patients with LQT2 with HERG mutations appear to be particularly sensitive to auditory stimuli. $^{7}$

We recently reported that heart rate responses and QT dynamics resulting from acute physical exercise and subsequent recovery differ in controls, LQT1 patients, and LQT2 patients. During a bicycle ergometer test, LQT1 patients, but not LQT2 patients, showed a pronounced reduction in the maximal heart rate achieved compared with control subjects. ${ }^{8}$
However, thus far there are no experimental data on the effects of mental stress on QT dynamics in patients with different types of inherited LQTS. Such information would be important in view of behavioural counselling of patients with LQTS with differing genotypes and their family members. Previously, QT interval has been found to shorten to a similar extent in response to mental and physical stress in non-LQTS patients with a fixed heart rate. ${ }^{9}$ We decided to investigate the effect of mental stress on heart rate responses, QT intervals, and QT interval dynamics, in LQT1 and LQT2 patients as well as in healthy controls. We used validated tests to elicit mental stress. ${ }^{10}$

\section{Methods}

PATIENT POPULATION

The study groups comprised 16 subjects with a documented KCNQ1 mutation, 14 subjects with an established HERG mutation, and 14 healthy control subjects. The LQT1 patient group consisted of five men and 11 women (age range 13-51 years), while the LQT2 group was composed of eight men and six women (16-59 years). The mean (SD) ages in these groups were 43 (10) years (controls), 35 (13) years (LQT1) and 41 (12) years (LQT2). The following criteria were required of the LQTS patients for acceptance into the study: a molecularly established mutation of the KCNQ1 or HERG gene, no history of syncope 
or cardiac arrhythmia, no history of organic or systemic disease other than LQTS, and no current medication known to affect cardiac function. The underlying mutations (with numbers of patients in parentheses) of the KCNQ1 potassium channel gene were as follows: G589D ( $n=12), G 269 S(n=2)$, and D $317 \mathrm{~N}(\mathrm{n}=2)$. The mutations of the HERG potassium channel gene were as follows: del453C $(\mathrm{n}=5)$, G584S $(\mathrm{n}=3), \mathrm{R} 176 \mathrm{~W}$ $(n=2)$, L552S $(n=2)$, P451L $(n=1)$, and G601S ( $n=1)$. The control group comprised eight men and six women aged 22-58 years who were not taking any medication known to affect cardiac function and without any evidence of cardiac disease.

The research protocol was reviewed and approved by the ethical review committee of the Department of Medicine, University of Helsinki, and informed consent was obtained from all study subjects.

CLINICAL METHODS

The patients were not allowed to eat, use tobacco products or drink anything except water during the four hours preceding the study. An intravenous cannula was inserted, and after 30 minutes of rest in the supine position, a resting 12 lead ECG was obtained. After this, a continuous ECG recording was begun and was continued until the end of the protocol.

After standardised instructions were provided by the study physician, the subjects performed a serial subtraction test, the Stroop colour-word test, and a serial addition test in a sitting position. ${ }^{11}$ These tests were performed back to back with no pauses in between except for instructions from the study physician. Before taking the serial subtraction tests and the three variations of the Stroop colour-word test, the patients were told that their performance would be compared with those of other study subjects. Twice, at preset points during each test, the subjects were told to increase speed. For the serial addition test, a prerecorded tape of sequential integers less than 10 was played to the patient, and the subjects were told to report the sum of two sequential integers to the study physician. The subjects were told that the number of mistakes would be compared with that of the other subjects. When the subjects' heart rate reached a maximum level more than three minutes from the start of the mental stress test, a second blood sample was taken.

When heart rates and blood pressures recovered to resting values after 5-10 minutes of rest in the supine position after mental stress testing, patients performed a bicycle ergometer test. The test was started at a workload of $30 \mathrm{~W}$, with a stepwise increase of $15 \mathrm{~W}$ every minute. When subjects' heart rate reached the maximum observed previously during mental stress, a third blood sample was taken. Each patient underwent both the mental stress test and a submaximal bicycle ergometer test.
ANALYSIS OF QT INTERVALS

QT intervals were measured at the maximum heart rate observed during mental stress and at a corresponding heart rate during exercise stress. QT intervals were measured from lead V3 at a paper speed of $50 \mathrm{~mm} / \mathrm{s}$ and an amplification of $0.1 \mathrm{mV} / \mathrm{mm}$. Lead $\mathrm{V} 3$ was used because it has the largest amplitude. ${ }^{12}$ ECG readers were blinded to the LQT status of the patients. The QT interval was measured from the onset of the QRS complex to the end of the $\mathrm{T}$ wave. The end of the QT interval was defined as the intersection of a tangent to the steepest downslope of the dominant repolarisation wave with the isoelectric line. Steady heart rate was determined at a point where heart rate had been constant for a minimum of 10 seconds. QT intervals at mental and exercise stress were measured from four consecutive QT intervals at this steady heart rate. QT interval corrected for heart rate (QTc) was determined according to Bazett's and Fridericia's formulas. We used the latter formula $\left(\mathrm{QT}_{\mathrm{cf}}\right)^{13}$ when analysing QT interval responses to stress stimuli because this formula may perform better at higher heart rates than the correction carried out according to Bazett. ${ }^{14}$ To evaluate QT adaptation to heart rate changes $(\Delta \mathrm{HR})$ during mental and exercise stress, $\Delta \mathrm{QT} / \Delta \mathrm{HR}$ values were also calculated by plotting change in QT duration against corresponding change in heart rate.

MEASUREMENT OF SERUM POTASSIUM AND PLASMA CATECHOLAMINE CONCENTRATIONS Blood samples for measurement of serum potassium and plasma catecholamine concentrations were taken after 30 minutes of rest in the supine position before mental stress, at the highest heart rate achieved during mental stress, and at a corresponding heart rate during physical stress. Concentrations of noradrenaline (norepinephrine) and adrenaline (epinephrine) in plasma were measured using an assay described elsewhere. ${ }^{15}$

\section{STATISTICAL ANALYSIS}

Continuous variables were expressed as mean (SD). For analysis of differences between groups, analysis of variance and MannWhitney tests were used as well as Student's independent means $t$ test. Spearman's correlation coefficient test was used to assess correlations between variables. A probability value of $\mathrm{p}<0.05$ was judged to be significant. SPSS software (SPSS Inc, Chicago, USA) was used for data analysis.

\section{Results}

HEART RATE RESPONSES DURING THE MENTAL AND EXERCISE STRESS TESTS

There were no significant differences in the resting or maximal heart rates achieved during the mental stress tests between the two groups of LQTS patients and the control group (fig 1). Heart rate responses from rest to mental stress and from rest to exercise stress were significant 

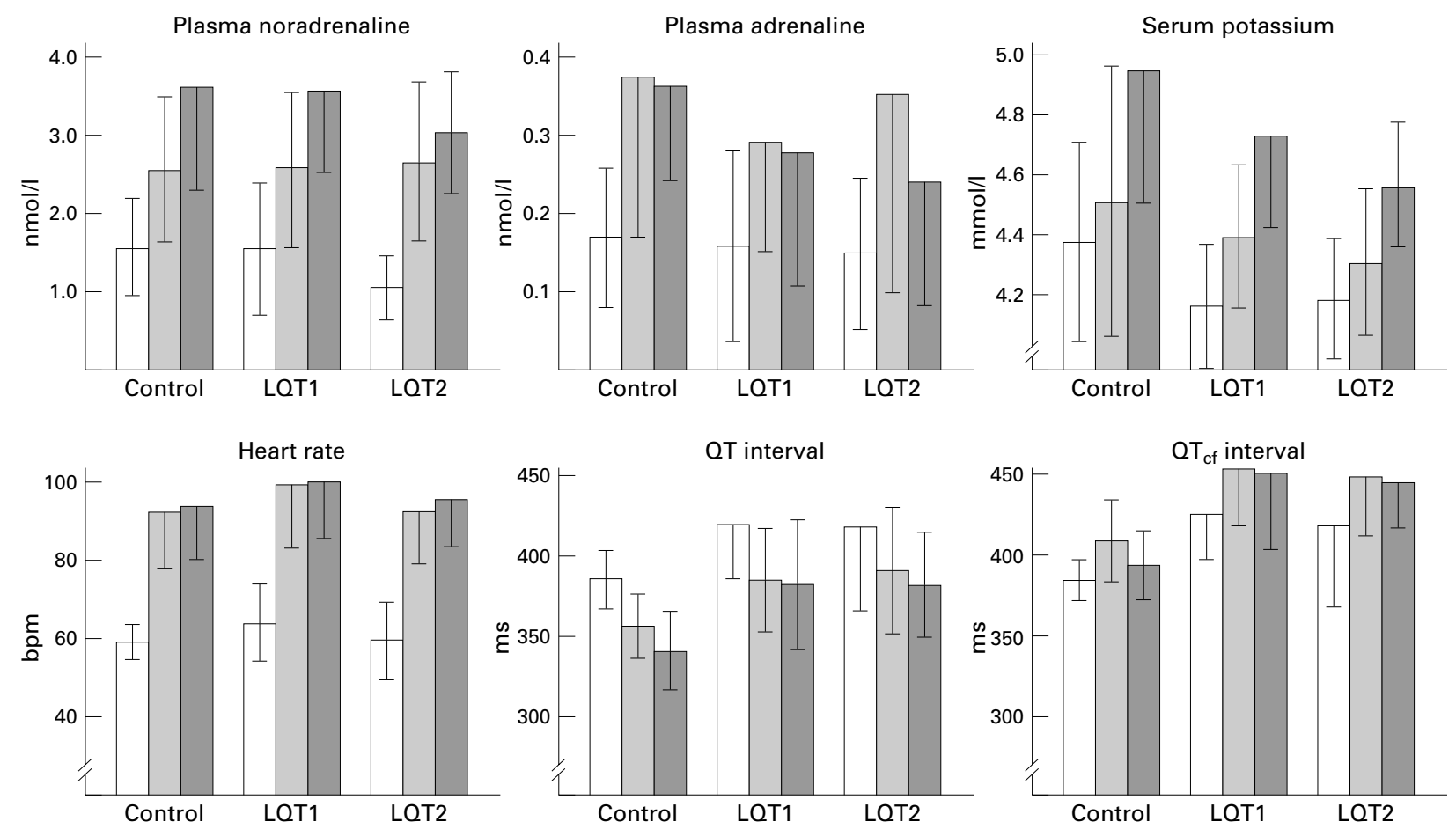

Figure 1 Plasma noradrenaline, plasma adrenaline, serum potassium, heart rate, $Q T$ interval, and $Q T$ interval adjusted according to Fridericia's formula ( $Q T_{c}$ ) in controls, patients with long QT syndrome type 1 (LQT1), and patients with long QT syndrome type 2 (LQT2) at rest (blank), and during mental stress (light grey) and exercise (dark grey).

in all three subject groups $(\mathrm{p}<0.001)$. While the mean heart rate changes from rest to mental stress were not significantly different in the LQT1 (36 (12)), the LQT2 (32 (9)), and the control group (31 (15)), subjects in each group showed pronounced interindividual differences in reactivity to mental stress. As a result, the corresponding ranges for the these changes were $10-65,17-63$, and $14-48$ in the three study groups, respectively (table 1 ).

RESULTS OF SERUM POTASSIUM AND PLASMA CATECHOLAMINE MEASUREMENTS

Mental stress did not significantly increase serum potassium concentrations in any of the groups studied, whereas physical exercise resulted in a significant $(p<0.05)$ increase in serum potassium concentrations in all three groups. The mean potassium concentrations achieved during physical exercise did not differ in the LQT1 patients $(4.7(0.3) \mathrm{mmol} / \mathrm{l})$,
LQT2 patients $(4.5(0.2) \mathrm{mmol} / \mathrm{l})$, or controls (4.8 (0.5) $\mathrm{mmol} / \mathrm{l}$ ) (fig 1 ).

Baseline plasma adrenaline and noradrenaline concentrations were similar in the three study groups. Whether assessed between rest and mental stress or between rest and physical stress, there was a highly significant $(\mathrm{p}<0.001)$, approximately $2-2.5$-fold increase in both plasma adrenaline and noradrenaline concentrations in all three subject groups. There was a tendency towards greater increases in plasma adrenaline concentrations during mental stress and greater plasma noradrenaline concentrations during physical stress (fig 1).

EFFECT OF MENTAL AND PHYSICAL STRESS ON QT INTERVAL

The QTc intervals at rest, calculated according to Bazett, were 429 (31) ms in LQT1 patients, 417 (43) $\mathrm{ms}$ in LQT2 patients, and 383 (13) $\mathrm{ms}$ in controls. As expected, the LQT1

Table 1 Heart rate and QT interval dynamics in control subjects, and patients with long QT syndrome types 1 (LQT1) and 2 (LQT2) at rest, and during mental stress and exercise

\begin{tabular}{|c|c|c|c|c|c|c|c|}
\hline & $\begin{array}{l}\text { Heart rate } \\
\text { (beats/min) }\end{array}$ & $Q T$ interval (ms) & $\triangle Q T$ interval (ms) & $\begin{array}{l}\text { Change from } \\
\text { resting values (\%) }\end{array}$ & $\Delta Q T / \Delta H R(\mathrm{~ms} / \mathrm{s})$ & $Q T_{c f}(m s)$ & $\Delta Q T_{c f}(m s)$ \\
\hline \multicolumn{8}{|l|}{ Control } \\
\hline Rest & $59(4)$ & $387(16)$ & - & - & - & $384(12)$ & - \\
\hline Mental stress & $90(15)$ & $358(22)^{\star \star \star \star \star}$ & $-29(25)^{\star \star}$ & $7.4(6.4)^{\star \star \star \star \star}$ & $-0.96(1.02)^{\star}$ & $409(25)^{\star \star \star}$ & $24(26)^{\star \star \star}$ \\
\hline Exercise stress & $93(13)$ & $341(25)^{\star \star \star \star \star}$ & $-47(31)^{\star \star}$ & $11.7(7.7)^{\star \star \star \star}$ & $-1.33(0.69)^{\star}$ & $393(21)^{\star \star \star}$ & $9(27)^{\star \star \star}$ \\
\hline \multicolumn{8}{|l|}{ LQT1 } \\
\hline Rest & $64(10)$ & $420(35)$ & - & - & - & $426(28)$ & - \\
\hline Mental stress & $98(16)$ & $386(33)$ & $-34(34)$ & $9.1(8.6)$ & $-1.21(1.19)$ & $453(35)$ & $27(36)$ \\
\hline Exercise stress & $100(14)$ & $382(41)$ & $-38(33)$ & $10.2(8.74)$ & $-1.33(1.27)$ & $451(48)$ & $25(42)$ \\
\hline \multicolumn{8}{|l|}{ LQT2 } \\
\hline Rest & $59(10)$ & $422(40)$ & - & - & - & $419(40)$ & - \\
\hline Mental stress & $91(13)$ & $392(40)$ & $-30(51)$ & $6.4(12.4)$ & $-0.71(1.68)$ & 449 (37) & $30(47)$ \\
\hline Exercise stress & $95(13)$ & $383(33)$ & $-38(46)$ & $8.5(10.9)$ & $-0.85(1.37)$ & $445(27)$ & $26(42)$ \\
\hline
\end{tabular}

Values are mean (SD).

${ }^{\star} \mathrm{p}=0.03,{ }^{\star \star} \mathrm{p}=0.04,{ }^{\star \star \star} \mathrm{p}=0.05,{ }^{\star \star \star \star} \mathrm{p}=0.005$, all others not significant.

$\mathrm{HR}$, heart rate; $\mathrm{QT}_{\mathrm{cf}}$, QT interval adjusted according to Fridericia's formula. 
and LQT2 patients had significantly longer QT intervals at rest, and during mental stress and exercise stress compared with control subjects (all p < 0.001) (table 1). In every group, QT intervals during mental stress and QT intervals during exercise were significantly correlated with each other $(p<0.01)$. A similar correlation was also evident for the corresponding $\mathrm{QTC}_{\mathrm{f}}$ values for each group studied $(\mathrm{p}<0.05)$ (table 1).

In healthy controls, shortening of the QT interval from rest to exercise $(\Delta \mathrm{QT})$ was more pronounced $(-47 \mathrm{~ms})$ than the corresponding shortening of the QT interval from rest to mental stress $(-29 \mathrm{~ms}, \mathrm{p}<0.01)$. In contrast, the QT interval response to exercise stress did not differ from that during mental stress in either LQT1 or LQT2 patients (table 1). The control group also showed further adaptation to exercise stress when this adaptation was calculated as percentage decreases from rest to mental stress or from rest to exercise $(\mathrm{p}<0.01)$.

In each group, the mean $\mathrm{QT}_{\mathrm{cf}}$ values during mental stress or during exercise were significantly shorter than at rest $(\mathrm{p}<0.001)$. In controls, $\mathrm{QT}_{\mathrm{cf}}$ intervals during exercise stress were significantly shorter than those during mental stress $(\mathrm{p}<0.05)$ (table 1$)$.

LQT1 patients, LQT2 patients, and controls did not differ significantly from each other in their QT interval adaptation to mental stress or to exercise stress. Control subjects showed a steeper $\Delta \mathrm{QT} / \Delta \mathrm{HR}$ slope during exercise than during mental stress $(\mathrm{p}<0.05)$ (table 1$)$.

When data at rest and during exercise were compared, changes in serum potassium concentration from rest to exercise were inversely correlated with changes in QT intervals in healthy controls (Pearson correlation coefficient $-0.828, \mathrm{p}<0.01$ ), but not in LQT1 and LQT2 patients (data not shown). No such correlation was found between changes in serum potassium concentrations associated with mental stress and $\Delta \mathrm{QT}$ values in any subject group. Changes in plasma catecholamine concentrations were not related to any significant extent to $\Delta \mathrm{QT}$ values in any subject group at any time.

\section{Discussion}

This report delineates experimental conditions under which the effects of mental stress on QT dynamics were investigated. Our data suggest that at similar heart rates, QT interval shortening in response to physical exercise is normally more pronounced than the corresponding response to mental stress, and that this capability of further shortening ventricular repolarisation in response to exercise stress is lost in both LQT1 and LQT2 types of LQTS.

In the present study the effects of mental and exercise stress on the QT interval were compared at similar heart rates. The intensity of the exercise test was adjusted according to individual heart rate increases during the preceding mental stress tests and, therefore, ECG artefacts during measurement of QT intervals were negligible. The pronounced increases in serum potassium concentrations during the modified bicycle ergometer test probably resulted from release of potassium ions from contracting skeletal muscles and reflects a considerable intensity of exercise. ${ }^{16}$

There was a slight although non-significant propensity towards a higher adrenaline and lower noradrenaline response during mental stress test than during exercise (fig 1). A similar finding was reported by Goldberg and colleagues. ${ }^{17}$ The adequacy of our design in causing severe mental stress is further supported by the finding that catecholamine responses during the mental stress test were similar in magnitude to those during exercise (2-2.5fold). Our study design for mental stress brought about mean heart rate increases of 31-34 beats/min in the three subject groups (table 1). In previous studies in which the effect of mental stress on cardiac function has been studied by laboratory experimentation, mean heart rate responses have typically varied between $8-20$ beats $/ \mathrm{min},{ }^{18-20}$ which further strengthens the assumption that our model was appropriate. In this study the diminished chronotropic response to maximal physical exercise typical of LQT1 patients was not observed, ${ }^{8}$ apparently because we used a submaximal exercise test.

Except for anecdotal findings in ambulatory recordings of individual patients, there appear to be no previous data on the effects of mental stress on QT dynamics in LQTS. Significant shortening of the QT interval was noted in patients with high degree atrioventricular block when they were subjected to a mental stress test, whether tested under conditions during which the ventricular rate was kept constant or it was permitted to increase. ${ }^{9}$ During the latter conditions, QT shortening during physical stress (66 ms or $15.5 \%$ ) was more pronounced than during mental stress (17 ms or $4 \%$ ), which is in accordance with our data. However, in our study, heart rate and therefore QT responses to mental stress were more pronounced. Hedman and Nordlander, ${ }^{9}$ as we did, observed striking interindividual variations in heart rate and QT responses to mental stress.

No arrhythmias occurred during the two test procedures in patients or controls. All LQTS patients were unequivocally affected by the LQTS as seen by molecular evidence of mutations in the KCNQ1 or HERG gene and by a prolonged QTc interval. We emphasise, however, that because of ethical objections we studied only asymptomatic gene carriers, which may generate a selection bias. In addition, we avoided acute startling stimuli such as loud auditory impulses, which may be particularly important neurogenic triggers for arrhythmia.

Although a significant shortening of QT interval in response to mental stress was observed in healthy controls as well as in LQT1 and LQT2 patients, QT interval shortening in response to exercise stress was greater in controls. In the latter patients, these changes in QT interval were significant when the calculations were based on $\Delta \mathrm{QT}$ intervals, percentages of resting QT intervals, or $\Delta \mathrm{QT}$ intervals 
adjusted to $\Delta \mathrm{HR}$. In the present study, $\Delta \mathrm{QT}$ adapted to $\triangle \mathrm{HR}$ was a realistic indicator of QT interval change because resting QT intervals and heart rates achieved during mental and exercise stress were somewhat different in controls, LQT1 patients, and LQT2 patients.

During cardiac repolarisation, KCNQ1 coassembled with MinK is responsible for the cardiac slowly activated inward rectifier potassium current $\left(\mathrm{I}_{\mathrm{Ks}}\right)$, while HERG, possibly complexed with MiRP $1,{ }^{6}$ accounts for the rapidly activated current $\left(\mathrm{I}_{\mathrm{kr}}\right) .{ }^{21} \mathrm{I}_{\mathrm{Ks}}$ is both activated and inactivated more slowly than $\mathrm{I}_{\mathrm{Kr}}$, which in turn is activated slowly but inactivated very rapidly. ${ }^{21}{ }^{22}$ In view of these electrophysiological differences of these two currents, it is somewhat surprising that we did not see any significant differences in the responses of the LQT1 and LQT2 patients to either physical or mental stress. The amount of $\mathrm{I}_{\mathrm{Kr}}$ has been shown to increase concomitantly with increases in extracellular potassium in vitro, ${ }^{23}$ even though potassium flow from the cardiac myocyte during repolarisation is outward and increased extracellular potassium should decrease the driving force for outward current through delayed rectifier potassium channels. There are insufficient data on the effects of extracellular potassium ions on $\mathrm{I}_{\mathrm{Ks}}$ in vivo but on the basis of in vitro studies, it has been suggested that $\mathrm{I}_{\mathrm{Ks}}$ decreases as extracellular potassium concentration is increased. ${ }^{24}$ Intravenous potassium has been shown to decrease QT and QTc intervals in patients with mutations in the HERG potassium channel ${ }^{25}$ and in patients with drug induced QT interval lengthening. ${ }^{26}$

In view of the above earlier findings, it is tempting to speculate that extracellular potassium concentration may have had some role in modifying QT responses to exercise stress. Changes in serum potassium concentrations from rest to exercise stress correlated with changes in QT interval from rest to exercise stress in healthy controls but not in LQT1 and LQT2 patients. Provided that wild type HERG and possibly KCNQ1 channels are more sensitive to extracellular potassium ions than mutated channels, a rise in potassium concentration during the transition from mental stress to exercise may induce a further shortening of the QT interval in healthy people but not in LQTS patients with defective potassium channels.

\section{STUDY LIMITATIONS}

All study subjects were aware of the study protocol before participating in the experimental procedures, and no unexpected, sudden, or startling stimuli were employed. It is possible that more abrupt or frightening triggers would have caused more pronounced changes in heart rate or QT dynamics. In addition, our inclusion criteria stipulated that only asymptomatic patients with genetically proven LQTS be recruited. Our study was carried out soon after genetic diagnosis of LQTS, facilitating clinical investigations before any interfering drug treatment. Similar studies after cessation of antiarrhythmic drug administration to symptomatic patients were considered unethical. The possibility thus remains that symptomatic gene carriers differ from the asymptomatic ones by some important mechanism that determines susceptibility to the stimuli that were used in the present study.

CONCLUSIONS

The present data indicate that QT adaptation to mental stress in healthy subjects and patients with LQTS is different: as studied by stress tests promoting similar heart rate accelerations, QT adaptation is more sensitive to physical than mental stress in healthy people. No such diverging pattern is seen in LQTS patients, at least in the asymptomatic ones.

We thank Hanna Ranne RN, for her expert help with the execution of the study protocol. This study was supported by grants from the Medical Council of the Finnish Academy, The Finnish Foundation for Cardiovascular Research, The Sigrid Juselius Foundation, and The Instrumentarium Science Foundation.

1 Romano C, Gemme G, Pongiglione R. Aritmie cardiache rare dell'eta pediatrica. Clin Pediatr (Bologna) 1963;45:656-83.

2 Schwartz PJ, Periti M, Malliani A. The long QT syndrome. Am Heart $\mathcal{f} 1975 ; 89: 378-90$.

3 Jervell A, Lange-Nielsen F. Congenital deaf mutism, functional heart disease with prolongation of the QT interval and sudden death. Am Heart f 1957;54:59-68.

4 Ward OC. A new familial cardiac syndrome in children. $\mathcal{F}$ Irish Med Assoc 1964;54:103-6.

5 Priori SG, Barhanin J, Hauer RNW, et al. Genetic and molecular basis of cardiac arrhythmias. Impact on clinical molecular basis of cardiac arrhythmias. Im

6 Abbott GW, Sesti F, Splawski I, et al. MiRP1 forms IKr potassium channels with HERG and is associated with car-
porith potassium channels with HERG and is

7 Schwartz PJ, Priori SG, Spazzolini C, et al. Genotypephenotype correlation in the long QT syndrome. Genespecific triggers for life-threatening arrhythmias. Circulation 2001;103:89-95.

8 Swan H, Viitasalo M, Piippo K, et al. Sinus node function and ventricular repolarization during exercise stress test in long QT syndrome patients with KvLQT1 and HERG potassium channel defects. F Am Coll Cardiol 1999;34: 823-9.

9 Hedman A, Nordlander R. Changes in QT and Q-aT intervals induced by mental and physical stress with fixed rate and atrial triggered ventricular inhibited cardiac pacing. PACE 1988;11:1426-31.

10 Streptoe A, Vogele C. Methodology of mental stress testing in cardiovascular research. Circulation 1991;83:II14-24.

11 Stroop JR. Studies of interferences in serial verbal reactions. Stroop JR. Studies of interferences
J Exp Psychol 1935;18:643-62.

12 Cowan JC, Yusoff K, Moore M, et al. Importance of lead selection in QT interval measurement. Am f Cardiol 1988; 61:83-7.

13 Fridericia LS. Die Systolendauer im Elektrokardiogramm bei normalen Menschen und bei Herzkranken. Acta Med Scand 1920;53:469-86.

14 Karjalainen J, Viitasalo $M$, Mänttäri $M$, et al. Relation between QT intervals and heart rates forom 40 to 120 beats/min in rest electrocardiograms of men and a simple method to adjust QT interval values. $7 \mathrm{Am}$ Coll Cardiol 1994;23:1547-53.

15 Scheinin M, Karhuvaara S, Ojala-Karlsson P, et al. Plasma 3,4-dihydroxyphenylglycol (DHPG) and 3-methoxy-4hydroxyphenylglycol (MHPG) are insensitive indicators of $\alpha_{2}$-adrenoceptor mediated regulation of norepinephrine release in healthy human volunteers. Life Sci 1991;49:7584 .

16 Lindinger MI. Potassium regulation during exercise and recovery in humans: implications for skeletal and cardiac recovery in humans: implications for skeleta

17 Goldberg AD, Becker LC, Bonsall R, et al. Ischemic, hemodynamic, and neurohormonal responses to mental and exercise stress experience from the psychophysiological investigations of myocardial ischemia study (PIMI). Circulation 1996;94:2402-9.

18 Hunziker PR, Gradel C, Muller-Brand J, et al. Improved myocardial ischemia detection by combined physical and mental stress testing. Am $\mathcal{F}$ Cardiol 1998;82:109-13.

19 Middlekauff HR, Nguyen AH, Negrao CE, et al. Impact of acute mental stess on sympathetic nerve activity and regional blood flow in advanced heart failure: implications for "triggering" adverse cardiac. Circulation 1997;96:1835-

20 Kral BG, Becker LC, Blumenthal RS, et al. Exaggerated reactivity to mental stress is associated with exerciseinduced myocardial ischemia in an asymptomatic high-risk induced myocardial ischemia in an asymp

21 Sapulation. Circulation 1997;96:4246-53. link between an inherited and an acquired cardiac arrhythmia: HERG encodes the Ikr potassium channel. Cell 1995;81:299-307. 
22 Sanguinetti MC, Curran ME, Zou A, et al. Coassembly of K(V)LQT1 and minK (IsK) protein to form cardiac I(Ks) K(V)LQT1 and minK (IsK) protein to for

23 Yang T, Roden DM. Extracellular potassium modulation of drug block of Ikr. Circulation 1996;93:407-11.

24 Sanguinetti MC, Jurkiewicz NK. Role of external Ca2+ and $\mathrm{K}+$ in gating of cardiac delayed rectifier $\mathrm{K}+$ currents. Pflugers Arch 1992;420:180-6.
25 Choy AM, Lang CC, Chomsky DM, et al. Normalization of acquired QT prolongation in humans by intravenous potassium. Circulation 1997;96:2149-54

26 Compton SJ, Lux RL, Ramsey MR, et al. Genetically defined therapy of inherited long-QT syndrome. Correction of abnormal repolarisation by potassium. Circulation 1996;94:1018-22.

\section{Combined pacing and percutaneous closing device therapy for dilated cardiomyopathy and patent ductus arteriosus}

A 64 year old woman was admitted to our unit for dyspnoea. Her chest radiograph (left panel) showed pronounced cardiomegaly. Physical examination revealed continuous machinery murmur over the infraclavicular area and her condition was confirmed to be patent ductus arteriosus (PDA) by echocardiography. Her ECG showed left bundle branch block (LBBB) with QRS duration of $180 \mathrm{~ms}$. Her left ventricular ejection fraction was $26 \%$. The pulmonary to systemic shunt ratio was 2.0 as determined by cardiac catheterisation; coronary arteries were normal. Percutaneous transcatheter closure of PDA was performed as the PDA might have contributed significantly to her heart failure. However, despite PDA closure and aggressive medical treatment, the patient remained in an inotrope dependent heart failure state. In view of LBBB and poor left ventricular function, a permanent biventricular pacemaker was implanted one week after PDA closure. Her heart failure improved following implantation of the pacemaker and she was weaned off inotropic support two days later.

Chest radiography (middle panel) was repeated two months after discharge from hospital. The cardiothoracic ratio was reduced when compared to the pre-implantation radiograph. Her functional class was improved to New York Heart Association class II and her left ventricular ejection fraction was 33\% one month after the procedures. The lateral chest radiograph (right panel) shows the positions of the atrial and two ventricular leads. The chest radiographs illustrate the dramatic reduction in cardiothoracic ratio before and after the procedures and the potential remodelling effect of biventricular pacemaker in patients with LBBB and poor left ventricular function.
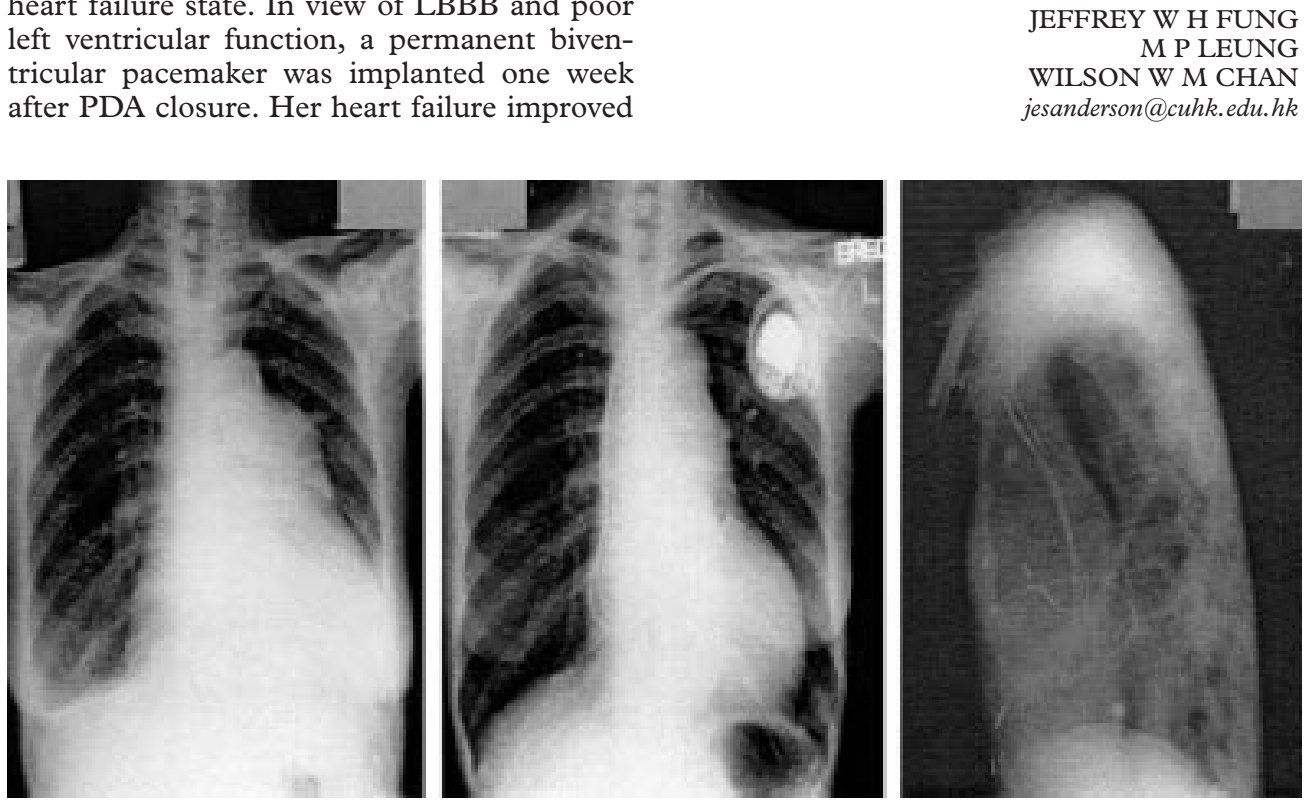